\title{
Coordination of Sucking, Swallowing, and Breathing and Oxygen Saturation During Early Infant Breast-feeding and Bottle-feeding
}

\author{
EUGENE C. GOLDFIELD, MICHAEL J. RICHARDSON, KIMBERLY G. LEE, AND STACEY MARGETTS
}

\author{
Children's Hospital Boston [E.C.G., S.M.], Boston, Massachusetts 02215; Harvard Medical School [E.C.G., K.G.L.], Boston, \\ Massachusetts 02215; Center for the Ecological Study of Perception and Action [M.J.R.], University of Connecticut, Storrs, \\ Connecticut 06269; Beth Israel Deaconess Medical Center [K.G.L.], Boston, Massachusetts 02215
}

\begin{abstract}
This prospective study compared the coordination of sucking, swallowing, and breathing and its relationship to oxygen saturation in infants during breast-feeding and bottle-feeding. After 4 to $6 \mathrm{wk}$ of exclusive breast-feeding, infants began bottle-feedings of expressed human milk using one of two systems: a soft-walled bottle and nipple (system 1, Playtex) or a hard-walled bottle and nipple (system 2, Avent). Infants' sucking, swallowing, breathing, and oxygenation were measured during breast-feeding and bottle-feeding, and coordination of these activities during breast-feeding and bottlefeeding were compared. During breast-feeding, swallowing occurred nonrandomly between breaths and did not interfere with breathing. The same distribution of swallowing occurred in infants fed with system 1, while swallowing occurred randomly in infants fed with system 2. Swallowing significantly increased during bottle-feeding among infants using system 2 , but decreased among infants using system 1. Infants using system 2 also had a greater instability in the coordination of sucking, swallowing, and breathing and more perturbation of breathing. Oxygen saturation was significantly higher in infants fed with system 1 compared with system 2 . These results suggest that the overall feeding pattern and oxygenation of system 1 are closer to the physiologic norm than system 2. (Pediatr Res 60: 450-455, 2006)
\end{abstract}

$\mathrm{I}^{\mathrm{n}}$ nfant feeding is a complex process, requiring the precise coordination of sucking, swallowing, and breathing. The pharynx is the shared anatomic pathway for both swallowing and breathing; however, these two activities are mutually exclusive. Therefore, the pharynx must be continually reconfigured so that an infant can successfully eat and breathe at the same time (1-6), but how infants coordinate these activities is largely unknown. There may also be differences in the overall coordination of these tasks during breast-feeding compared with bottle-feeding, but this has not yet been explicitly determined. Unlike artificial bottle nipples, the human breast is compliant in response to an infant's suckling activity, and the elasticity of the breast nipple allows it to transform to fit the shape, size, and positioning of the infant's mouth (7). In addition, the magnitude and consistency of the milk flow from

Received January 6, 2006; accepted May 22, 2006.

Correspondence: Eugene C. Goldfield, PhD, Children's Hospital Boston, 300 Longwood Avenue, Boston, MA 02115; E-mail: Eugene.Goldfield@childrens.harvard.edu

This study was supported by a grant from Playtex Products, Inc. (Westport, CT), which also provided both types of infant bottles and nipples for the research. Supported by NIH grant 2R44HD047128 to the first author and by the Children's Hospital Boston Mental Retardation and Developmental Disabilities Research Center, \#P01 HD18655.

DOI: $10.1203 / 01 . p d r .0000238378 .24238 .9 d$ a milk bottle are quite different from that of the breast, and milk bottles may have the possibility of internal pressure, which provides resistance to infant suckling. There may also be distinct differences among different bottle-feeding systems (e.g. soft- versus hard-walled bottles, different nipples and venting systems).

One key difference between breast-feeding and bottlefeeding is oxygen saturation. Previous studies have consistently demonstrated that breast-fed babies have higher oxygen saturation than bottle-fed babies $(8-10)$. A frequently cited explanation for this difference is that bottle-feeding may promote a higher rate of swallowing and, in turn, more frequent interruptions of breathing (11). Indeed, studies have shown that there is less ventilatory disruption during breast-feeding compared with bottle-feeding $(10,12,13)$, which may result in higher oxygen saturation. This implies that the coordination of swallowing and breathing is intimately involved in blood oxygenation during feeding, but relatively few studies have examined the relationship between oxygen saturation and the coordination of sucking, swallowing, and breathing in healthy full-term infants.

The objective of our study was to compare the coordination of sucking, swallowing, and breathing during breast-feeding and bottle-feeding. We also examined the relationship between oxygen saturation and coordination. To allow a detailed analysis of these events, we recorded sucks, swallows, respiratory airflow, and respiratory movements and analyzed the location of swallows to compare coordination. We studied 1) the distribution of swallows relative to sucking and breathing, 2) the amount of swallowing, 3) the stability of swallowing, 4) oxygen saturation, and 5) the correlation between oxygen saturation and swallowing variability. Our hypotheses were that during breast-feeding, swallows would be distributed nonrandomly (i.e. at specific locations) and that a bottlefeeding system that more closely mimics the physiologic norm would promote a similar swallowing pattern. We also hypothesized that during periods of higher oxygenation, the coordination of sucking, swallowing, and breathing would be more stable (have lower variability) and that during periods of lower oxygenation, the variability of swallowing relative to breathing would be greater.

\section{METHODS}

Study participants. Mothers and infants were recruited in hospital on the second day after birth by research nurses at Beth Israel Deaconess Medical 
Center in Boston, MA. Each day during the recruitment period, one of two research-study nurses reviewed data from the birth census to determine eligibility of potential participants. Eligibility criteria included infant birth weight greater than $2500 \mathrm{~g}$, no medical problems that might influence feeding, and no maternal complications during pregnancy or delivery. All infants who met these criteria were eligible for the study regardless of gender or ethnicity. A second set of eligibility criteria concerned the mother's plans for feeding the infant. Only those women who indicated to the research nurse that they planned to breast-feed the infant exclusively for at least 4 to $6 \mathrm{wk}$ after birth before initiating bottle-feeding (while continuing to breast-feed) were eligible. Mothers were excluded if their medical charts indicated any structural or functional characteristics that might impede breast-feeding. The study was described, and mothers were told that study participation involved a laboratory visit at Children's Hospital Boston after 4 wk of exclusive breast-feeding. Women who met these criteria and agreed to participate provided written informed consent. Mothers agreed to be contacted by telephone approximately $4 \mathrm{wk}$ after leaving the hospital to arrange a time for the laboratory visit. If a mother decided to discontinue participation at any time before the laboratory visit, she and the baby were replaced in the study.

Study procedure. The research protocol was approved by the clinical investigation committees at Beth Israel Deaconess Medical Center and Children's Hospital Boston. Infants served as their own controls for comparison of breast-feeding and bottle-feeding. After 4 to $6 \mathrm{wk}$ of exclusive breast-feeding, mothers introduced an artificial nipple and bottle for supplemental feeding. All bottle-feeding was performed with expressed breast milk. For supplementation, mothers chose either system 1 (a Playtex bottle with a soft-liner that collapses during feeding and a NaturaLatch nipple; Playtex Products, Inc., Westport, CT) or system 2 (an Avent newborn bottle and nipple; Avent Ltd., Bensenville, IL); both bottle systems are commercially available and had equivalent flow rates. Within $2 \mathrm{wk}$ of initiating supplemental bottle-feeding, mothers and infants came to the Infancy Laboratory in the Department of Psychiatry at Children's Hospital Boston for the laboratory visit, which consisted of a 30-min procedure during which instrumented recording of 3 to 4 min of sucking, swallowing, breathing, and oxygen saturation during breast-feeding and bottle-feeding, respectively, were performed; the infant was weighed; and the mother completed a brief oral interview to determine her attitudes about breast-feeding.

For instrumented recordings of the infant's sucking, swallowing, and breathing during breast-feeding and bottle-feeding, sensors were attached to the skin of both the mother's breast and the infant. The goal was to use the same sensors for breast-feeding and bottle-feeding to get equivalent information. To record the intraoral sucking pressure during breast-feeding, a catheter was secured using medical-grade tape (Transpore Surgical Tape; 3M, St. Paul, $\mathrm{MN}$ ) so that its open end was aligned with the tip of the mother's nipple and was taken into the infant's mouth during feeding. To record intraoral pressure during bottle-feeding, the infant was fed with a nipple that was modified slightly from the ones used at home. A catheter identical to the one previously attached to the mother's breast was embedded within the bottle nipple so that it entered the infant's mouth during feeding. To record swallowing, a miniature wireless microphone (SC4-CT; Shure, Inc., Niles, IL) was secured with tape to the skin beneath the cricoid and oriented toward the pharynx (14). To record respiratory movements, inductance bands (Inductotrace respiratory monitors; Ambulatory Monitoring, Inc., Ardsley, NY) were secured around the infant's chest and abdomen, and an oxygen saturation sensor was also secured on one foot; the signal from the latter was analyzed by a pulse oximeter (Radical; Masimo Corporation, Irvine, CA). All sensor signals were digitized and simultaneously recorded with data-acquisition software (WinDaq/Pro+; Dataq Instruments, Inc., Akron, $\mathrm{OH}$ ) on a laptop computer (Dell Latitude CPi; Dell, Inc., Round Rock, TX). Infants were also weighed after feeding using a pediatric scale (Model 4802; Scaletronix, Carol Stream, IL).

Algorithms for locating swallows and calculating relative phase. To examine the temporal relationship among sucking, swallowing, and breathing, 10 consecutive samples of 5- to 10-s periods of breast-feeding and bottlefeeding behavior by each infant were extracted for analysis. These typically exhausted all the sucking behavior recorded, with periods of pause in sucking and/or the absence of swallowing excluded. The samples were saved as text files of the individual channels. Algorithms written in MATLAB (The MathWorks, Inc., Natick, MA) were used for Butterworth filtering (Besser Associates, Mountain View, CA) each recorded channel to calculate the relative phase between sucking and breathing, to calculate the location of swallows with respect to that relative phase, and to count the number of swallows. The results were then plotted as histograms in which each 360-degree cycle was divided into 20-degree bins, and the number of swallows in each bin was determined. If swallows were attracted to particular phase relations between sucking and breathing, swallows would be distributed nonrandomly.
Circular statistics. A statistical approach, termed circular or directional statistics, was used to determine whether the distribution of swallows was nonrandom (15-17). By recording the observed swallows in a table of their observed frequency across a range of values between zero and 180 degrees, we were able to determine a mean angle weighted by their relative distribution. Circular statistics may be preferable to other nonparametric methods because the result can be interpreted directionally (i.e. spatially). A standard nonparametric test would allow us to reject the null hypothesis that swallows were uniformly distributed in phase space, but would not reveal where swallows were most dense. Other statistical tests for parametric comparisons of group data included $\chi^{2}$ analyses for frequency of swallows during breastfeeding and bottle-feeding, analysis of variance (ANOVA) to analyze amount and stability of swallowing, and categorized correlational analyses of oxygen saturation by group and bottle condition.

\section{RESULTS}

Subject demographics. A total of 70 infants were enrolled in the study. Of these 70 infants, 34 were unable to complete both the home and laboratory testing portions of the study: 15 mothers were not ready to discontinue exclusive breastfeeding $6 \mathrm{wk}$ after hospital discharge; mothers of another nine infants switched bottle-feeding systems after their original choice, which made them ineligible to continue on the study, and for 10 infants, we were unable to record sucking at the breast because the infant rejected the tube taped to the nipple when it was presented for feeding. Therefore, after attrition, the study population comprised the first 36 healthy newborns who were able to successfully complete both the transition from breast to bottle during a 4- to 6-wk period and laboratory testing. There was no difference in dropout rates between the two bottle-feeding groups. Infant characteristics are shown in Table 1. All infants were full term. Birth weights and weights at weeks 4 to 6 were similar in the two bottle-feeding groups. There were no significant differences between groups with regard to pacifier use.

Individual data. To illustrate the analyses depicting the location of swallows relative to sucking and breathing, we first present data from a single 5-s sample of feeding from an individual infant (Fig. 1). Figure 1A depicts a swallow signal indicating that the infant produced four successive swallows (the four sharp amplitude peaks) during this 5-s period. The swallow signal was then filtered by a MATLAB algorithm, rectified, and then superimposed onto the respiratory (solid line) and sucking (dashed line) signals (Fig. 1B). From this figure, it is evident that swallows occur at the peaks of intraoral sucking pressure (inverted in the figure to be the valley) and that during two of the four swallows, there is a flattening of respiratory amplitude (see the middle two swallows in Figure $1 B$ ). The four swallows of shown in Figure $1 A$ were then presented in polar coordinates to indicate their

Table 1. Baseline characteristics of participants

\begin{tabular}{lcc}
\hline & $\begin{array}{c}\text { Infants using system 1 } \\
(\mathrm{n}=18)\end{array}$ & $\begin{array}{c}\text { Infants using system 2 } \\
(\mathrm{n}=18)\end{array}$ \\
\hline Maternal age, $\mathrm{y}^{*}$ & $32.62(3.69)$ & $32.45(4.18)$ \\
Infant characteristics & & \\
No. of boys, $\mathrm{n}$ & 9 & 9 \\
Birth weight, $\mathrm{g}^{*}$ & $3395(540.78)$ & $3519(415.00)$ \\
Weight at test, $\mathrm{g}^{*}$ & $5097.62(718.18)$ & $5357.43(555.62)$ \\
Age at test, $\mathrm{d}^{*}$ & $45.69(7.93)$ & $43.21(7.72)$ \\
\hline
\end{tabular}

* Mean (SD). 

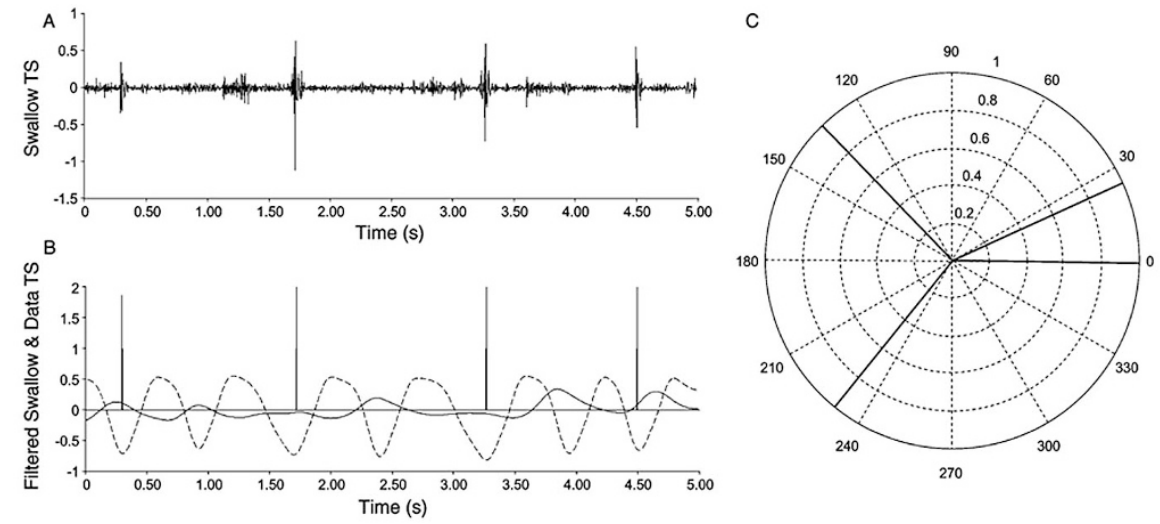

Figure 1. Coordination of swallowing relative to sucking and breathing in a single infant, as illustrated by the swallow signal $(A)$, a filtered and rectified swallow signal superimposed on the respiratory (solid line) and sucking (dashed line) waveforms $(B)$, and a polar plot of the cluster of swallows relative to the phase between sucking and breathing $(C)$. location in a 360-degree circular distribution of sucking and breathing (Fig. 1C). As can be seen in the figure, the four swallows are not randomly distributed, but rather tended to cluster near zero and around 180 degrees relative phase. These data thus suggest that swallows are not randomly distributed during feeding, but rather are likely to occur at particular locations in a space partitioned by ongoing sucking and breathing patterns.

Group data. Data from each group of infants (fed with system 1 or 2) were then examined and the distribution of swallowing relative to sucking and breathing during breastfeeding and bottle-feeding in each group was determined. Each 360-degree cycle of sucking and breathing was divided into 20-degree "bins," and the number of swallows in each bin was determined. Separate $\chi^{2}$ analyses were conducted on the observed and expected frequencies of swallows during breastfeeding (Fig. 2A) and bottle-feeding (Fig. 2B) in each group. As expected, swallowing was distributed nonrandomly during breast-feeding in both groups $(\times 2=32.75, d f=8, p<$ 0.0001 and $\times 2=22.42, d f=8, p<0.004$ for systems 1 and 2 , respectively). During bottle-feeding, swallowing was also distributed nonrandomly among infants using system $1(\times 2=$ 57.50, $d f=8, p<0.0001)$. However, infants using system 2 swallowed at random locations in the circular distribution ( $\times 2=9.138, d f=8, p=0.3307)$. Thus, when infants were switched from breast-feeding to bottle-feeding, only infants using system 1 continued to organize their sucking, breathing, and swallowing in a manner similar to their breast-feeding.

Inspection of these results suggest that there may be differences in the overall amount of swallowing in infants using the different bottle systems, so a parametric test was conducted on the data from the two groups. A group (system 1, system 2) by condition (breast-feeding, bottle-feeding) repeated-measures ANOVA indicated no significant main effects of group or condition, but did reveal a significant group by condition interaction, $\left(F_{1,68}=4.567, p=0.0362\right)$. Figure 3 depicts the mean number of swallows during breast- and bottle-feeding in each group. The post hoc analysis indicates that when infants switched from breast-feeding to bottle-feeding, there was a significant decrease in swallowing in infants using system 1 , but a significant increase in swallowing in infants using system 2 .

The above findings also suggest that the organization of swallowing in infants using system 2 may be more variable (i.e. less stable) than in those using system 1 . The measure of the SD of relative phase is indicative of the relative stability of coordination $(18,19)$, so we postulated that group differences in the SD of relative phase between systems 1 and 2 may shed light on differences in the variability of swallowing between the two groups. In particular, we hypothesized that system 1 may allow infants to continue swallowing with less perturbation of their breathing compared with system 2. An ANOVA on the SD of swallowing relative phase by infants in each of the two bottlefeeding groups during breast-feeding and bottle-feeding indicated a main effect of group $\left(\mathrm{F}_{1,68}=4.08, p=0.047\right)$, but no condition main effect or group by condition interaction (Fig. 4). Infants using system 2 exhibited significantly higher SD of swallowing relative phase and, therefore, greater instability in the coordination of sucking, breathing, and swallowing.

We then examined the relation between the stability of the coordination of swallowing, sucking, and breathing and blood oxygenation. First, we measured mean (Fig. 5A) and minimum (Fig. $5 B$ ) percentage of oxygen saturation during breastfeeding and bottle-feeding in infants using the two feeding systems. A group (system 1, system 2) by condition (breast-
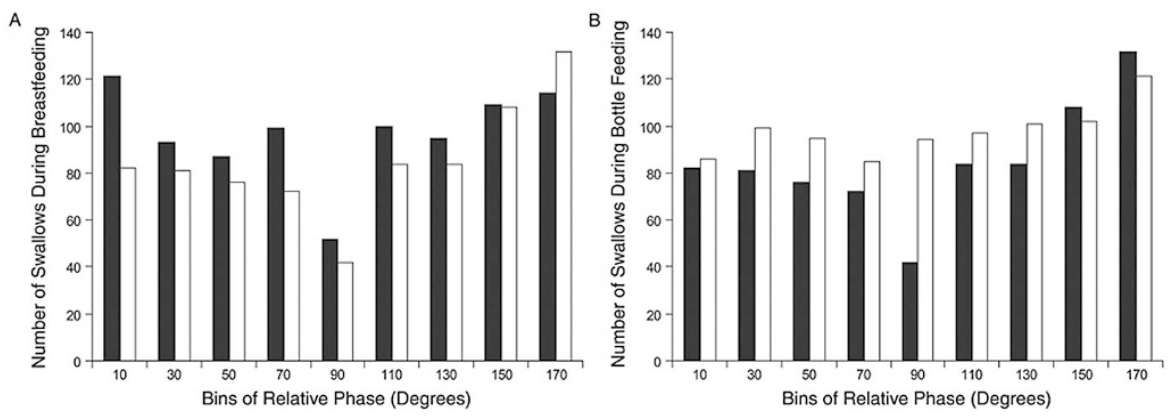

Figure 2. Frequency distribution of swallowing with respect to sucking and breathing during breastfeeding $(A)$ and bottle-feeding $(B)$ for infants fed with the two different feeding systems. $p<0.0001$ during breast-feeding and bottle-feeding for infants fed with system $1 ; p<0.004$ and $p=0.3307$ during breast-feeding and bottle-feeding for infants fed with system 2. Bottle system 1 (filled columns); bottle system 2 (open columns). 


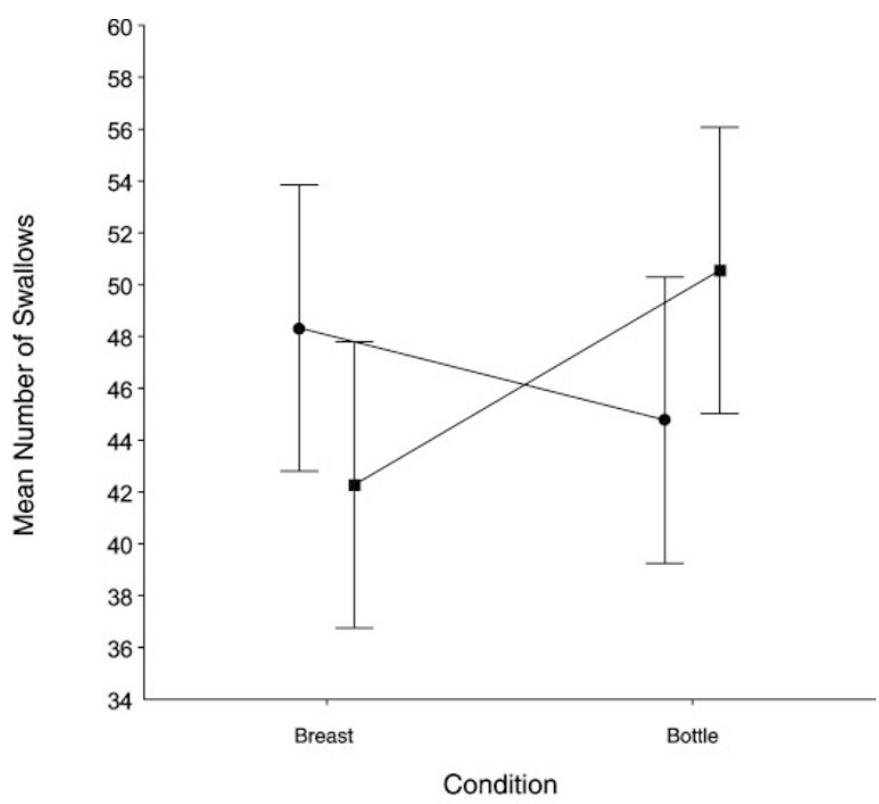

Figure 3. The mean number of swallows during breast-feeding compared with bottle-feeding for infants using the two different feeding systems. $p=$ 0.362. Bottle system $1(\mathbf{O})$, bottle system $2(\mathbf{\square})$.

feeding, bottle-feeding) ANOVA revealed group by condition interactions for both mean percentage of oxygen saturation $\left(F_{1,68}=6.70, p=0.011\right)$ and minimum percentage of oxygen saturation $\left(F_{1,68}=4.56, p=0.036\right)$. Post hoc analyses indicated that, in both cases, the significant interaction was due to the reduced oxygenation in infants using system 2 during bottle-feeding compared with their own breast-feeding. For infants using system 1, there were no differences in mean or minimum oxygen saturation during breast-feeding and bottle-feeding.

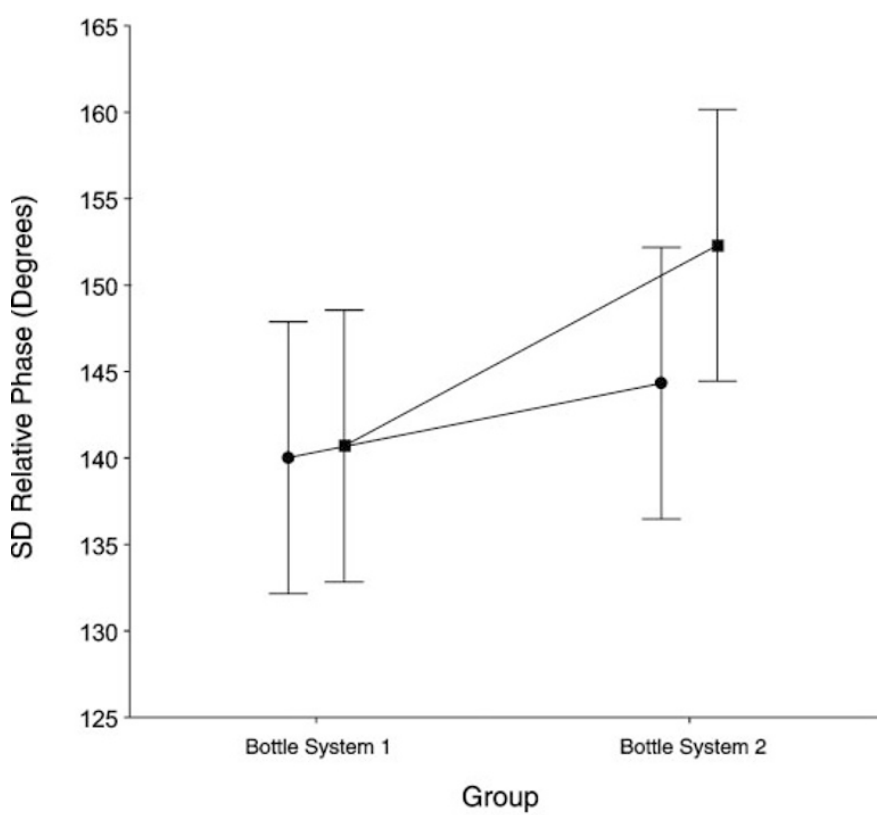

Figure 4. SD of swallowing relative phase with respect to sucking and breathing during breast- and bottle-feeding for infants using the two different feeding systems. $p=0.047$. Breast-feeding $(\mathbf{\bullet})$, bottle-feeding $(\boldsymbol{\square})$.
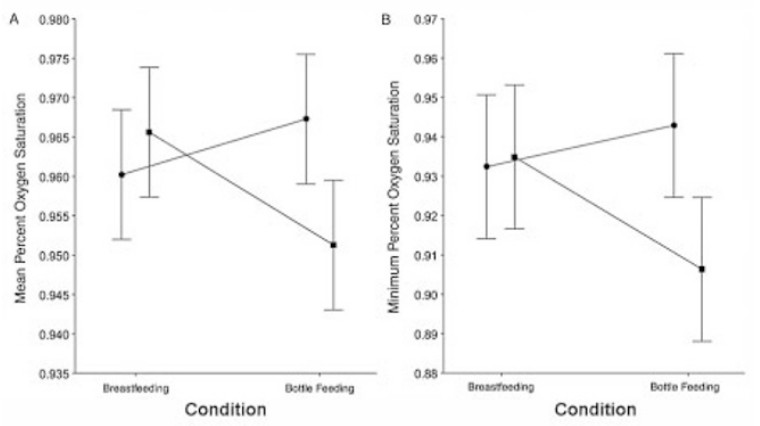

Figure 5. Mean $(A ; p=0.011)$ and minimum $(B ; p=0.036)$ oxygen saturation during breast- and bottle-feeding for infants using the two different feeding systems. Bottle system $1(\bullet)$, bottle system $2(\boldsymbol{\square})$.

To then determine whether the finding of lower oxygenation in the group using system 2 was related to the organization of their swallowing, we calculated categorized correlation coefficients between mean percentage of oxygenation and $\mathrm{SD}$ of swallowing relative phase separately by group and condition (Fig. 6). There were no significant correlations between oxygenation and SD of swallowing relative phase during breast-feeding or bottle-feeding in infants using system 1. However, lower mean percentages of oxygen saturation values were significantly correlated with higher SD of swallowing relative phase during bottle-feeding by infants using system $2(r=-0.4935, p=0.0374)$. Infants using system 2 thus had more variable swallowing and a correspondingly lower mean percent oxygen saturation.

\section{DISCUSSION}

This study examined the coordination of sucking, swallowing, and breathing during breast-feeding and compared it to coordination during bottle-feeding among infants using two different feeding systems. Our hypotheses were that during

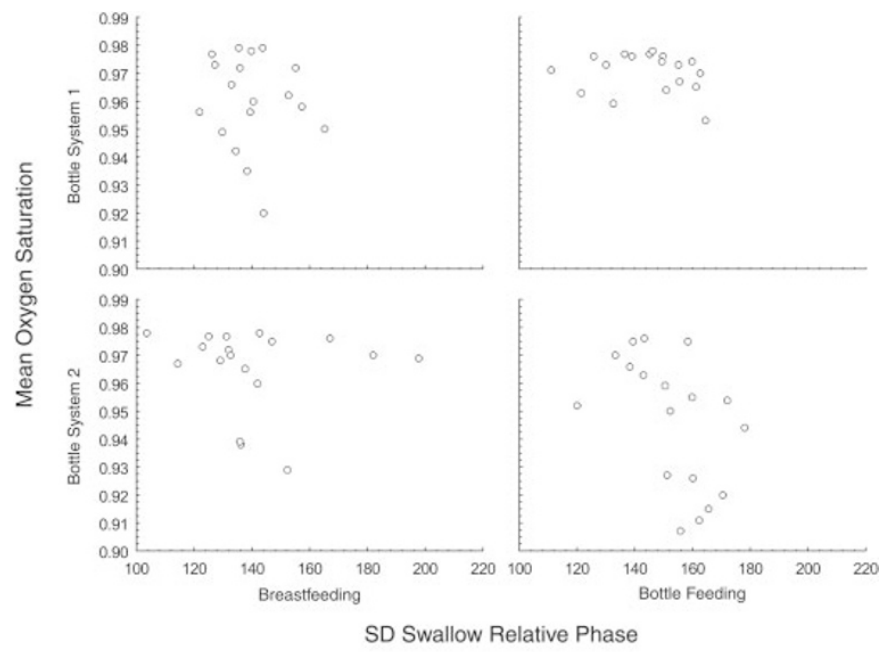

Figure 6. Correlation coefficients categorized by the relation between mean oxygen saturation for infants in each bottle system group and SD of swallowing relative phase with respect to sucking and breathing during breast- and bottle-feeding. $p=0.0374$. 
breast-feeding, swallows would be distributed nonrandomly (i.e. at particular locations) and that a bottle-feeding system that more closely mimics the physiologic norm would promote a similar swallowing pattern. We also compared oxygenation during both breast-feeding and bottle-feeding and hypothesized that during periods of higher oxygenation, the coordination of sucking, swallowing, and breathing would be more stable (have lower variability), and a bottle-feeding system that more closely mimics the physiologic norm of breast-feeding would more likely promote a coordination pattern with lower variability.

The study findings presented here demonstrate that during breast-feeding swallowing is segregated from breathing. These data suggest that sucking and breathing patterns create "windows of opportunity" for swallows and that the central nervous system may look for opportunities within ongoing sucking and breathing patterns in which to fit swallows, making it possible for an infant to continue feeding without interruption. Thus, infants are able to maintain a relatively constant milk flow and continue breathing by inserting swallows into particular regions of the extant sucking and breathing relationship. The results are consistent with and extend the findings of earlier studies $(3,4,20)$.

The results of this study also indicate that there were significant differences in coordination patterns in infants using different bottle-feeding systems. System 1 (Playtex) promoted a pattern of coordination between sucking, swallowing, and breathing that was more similar to the physiologic norm than was system 2 (Avent). Infants using system 1 distributed their swallows nonrandomly in time so that they were less likely to occur at the location of respiratory inspirations. By contrast, infants fed with system 2 distributed their swallowing more randomly, even when they segregated swallowing from breathing while they were breast-feeding. Furthermore, there were also statistically significant differences between the two systems in the amount of swallowing and in the stability of coordination of sucking, swallowing, and breathing. One possible consequence of this increased swallowing is that infants who swallow more frequently accumulate air in the stomach, which may cause postfeeding gastric upset. A relevant finding in this regard is reported in one of the few studies that have measured oxygen saturation during and after breast-feeding and bottle-feeding (21). The authors reported significantly lower oxygenation following bottle-feeding than during the actual feeding period and attribute this to burping and gastric distress after the feeding. A follow-up study that examines swallowing during feeding with pulse oximetry measures during and after feeding may help to clarify the postfeeding distress experienced by some bottle-fed infants.

Additionally, the reduced oxygenation (decreased oxygen saturation) that we observed during bottle-feeding compared with breast-feeding is consistent with previous studies $(8-10)$. Previous research suggests that alterations in blood oxygen levels are due to decreased ventilation $(20,22)$, which may be due to airway closure associated with swallowing and decreased ventilatory effort (20). Therefore, the higher oxygen levels that have been consistently observed during breastfeeding may be due to more coordinated sucking, swallowing, and breathing compared with bottle-feeding. This hypothesis is supported by our coordination analyses, which show the well-organized pattern of swallowing relative to sucking and breathing during breast-feeding. The coordination analyses also suggest that one reason why infants using system 1 exhibited similar oxygenation during both breast-feeding and bottle-feeding was that their swallowing was better organized.

The fact that oxygen saturation is higher during breastfeeding than bottle-feeding suggests something about the overall mechanics of the feeding process. There may be a mechanistic basis for the advantages of breast-feeding over bottle-feeding as a result of differences in tongue posture and less disruption of breathing. By extension, there may be differences among bottle-feeding systems, some of which may promote more natural postures and breathing patterns than others. If a system can be designed that promotes less swallowing, babies can feed more like the natural physiologic norm of breast-feeding.

What may have contributed to the variable location and instability of swallowing during feeding in the group using system 2? During breast-feeding, the tongue is grooved around the nipple and remains under the nipple throughout feeding $(2,7)$. The muscular action of the tongue produces peristalsis, so that the timing of swallowing is a continuous event in a sequence of standing waves (2). The position of the tongue during bottle-feeding in the two groups may have been different, but we do not know this with certainty. If the tongue is more "piston-like" in infants using system 2 and the task of finding the windows of opportunity for swallowing is more difficult, swallowing may occur at a moment in the respiratory cycle that destabilizes breathing, and, therefore, may promote oxygen desaturation. Ultrasound or other imaging studies in conjunction with our relative phase and oxygenation measures may help clarify this hypothesis.

A potential limitation of this study is that all bottle-feeding measurements were performed after breast-feeding. Since breast-feeding was "early" in the feeding cycle and bottlefeeding was "later," some of the differences in sucking patterns may be due to the relative timing during the feeding cycle. A cross-over study design (in which bottle-feeding measurements were also performed before breast-feeding) may thus have been beneficial in confirming these results. However, the way the study was performed mimics the reallife feeding patterns of infants (i.e. breast-feeding first, followed by a switch to a bottle) and therefore has external validity.

Guidelines from the American Academy of Pediatrics promote exclusive breast-feeding for the first 6 mo, with continuation for at least the first year (23). Although breast-feeding is clearly best for infants, it may not always be possible. To the extent that an artificial nipple and milk container allow the hungry infant to suck vigorously without unduly competing with breathing, the experience of bottle-feeding may be more similar to breast-feeding. By designing artificial nipples and reservoirs that are compatible with the intrinsic dynamics of infant behavior, it may be possible to make oral feeding by bottle more similar to the physiologic norm of breast-feeding. The artificial nipple used in system 1 in this study explicitly 
models the elasticity, shape, and texture of the human nipple, and the collapsible milk reservoir supports a constant milk flow that does not increase hydrostatic pressure as the remaining volume decreases. The nipple provides 1) a tip that extends elastically so that tongue peristalsis can more effectively draw milk into the posterior pharyngeal region to accumulate gradually before swallowing, 2) a shape that provides a rounded contour similar to the region between the nipple and areola of the breast, and 3) a textured region of the nipple contacted by the infant's lips that provides a slip-free surface to promote latching on with an effective seal between lips and the silicone material. The mechanical properties of the artificial nipple, like the breast, provide an elastic response in which the nipple extends and withdraws in a piston-like fashion, storing and releasing potential energy. Breast-feeding infants who have mastered a suck-swallow response that capitalizes on this stored potential energy may be able to slow their respiratory rate so that their swallowing fits the respiratory rhythm without prolonged interruptions.

In conclusion, the results of this study have several practical implications for evaluating issues of great concern to women who breast-feed but who may elect to supplement breastfeeding with breast milk fed by bottle and artificial nipple. These women face the difficult choice of which bottle and artificial nipple to use, and many do so in the context of confusing and conflicting professional and family advice. There has also been concern in the clinical literature regarding the potential for nipple confusion if artificial nipples are used. The methodology of the present study suggests a research strategy that may be used to compare the overall dynamics of breast-feeding and bottle-feeding under a range of parametric manipulations. It may thus be possible to use principles derived from the study of coordination dynamics to continue to improve ways to supplement breast-feeding when women and their families choose to do so.

\section{REFERENCES}

1. Bosma JF 1986 Anatomy of the Infant Head. Johns Hopkins University Press, Baltimore

2. Bosma JF, Hepburn LG, Josell SD, Baker K 1990 Ultrasound demonstration of tongue motions during suckle feeding. Dev Med Child Neurol 32:223-229

3. Gewolb IH, Vice FL, Schwietzer-Kenney EL, Taciak VL, Bosma JF 2001 Developmental patterns of rhythmic suck and swallow in preterm infants. Dev Med Child Neurol 43:22-27

4. Gewolb IH, Bosma JF, Reynolds EW, Vice FL 2003 Integration of suck and swallow rhythms during feeding in preterm infants with and without bronchopulmonary dysplasia. Dev Med Child Neurol 45:344-348

5. Reynolds EW, Vice FL, Bosma JF, Gewolb IH 2002 Cervical accelerometry in preterm infants. Dev Med Child Neurol 44:587-592

6. Selley WG, Ellis RE, Flack FC, Brooks WA 1990 Coordination of sucking, swallowing and breathing in the newborn: its relationship to infant feeding and normal development. Br J Disord Commun 25:311-327

7. Riordan J 2005 Breastfeeding and Human Lactation. Jones and Bartlett, Boston

8. Blaymore Bier J, Ferguson A, Morales Y, Liebling M, Oh W, Vohr B 1997 Breastfeeding infants who were extremely low birth weight. Pediatrics 100:e3

9. Chen CH, Wang TM, Chang HM, Chi CS 2000 The effect of breast- and bottlefeeding on oxygen saturation and body temperature in preterm infants. J Hum Lact $16: 21-27$

10. Meier P 1988 Bottle- and breast-feeding: effects on transcutaneous oxygen pressure and temperature in preterm infants. Nurs Res 37:36-41

11. Miller MJ, DiFiore JM 1995 A comparison of swallowing during apnea and periodic breathing in premature infants. Pediatr Res 37:796-799

12. Meier P, Pugh EJ 1985 Breast-feeding behavior of small preterm infants. MCN Am J Matern Child Nurs 10:396-401

13. Meier P, Anderson GC 1987 Responses of small preterm infants to bottle- and breast-feeding. MCN Am J Matern Child Nurs 12:97-105

14. Boiron M, Rouleau P, Metman EH 1997 Exploration of pharyngeal swallowing by audiosignal recording. Dysphagia 12:86-92

15. Batschelett E 1981 Circular Statistics in Biology. Academic Press, New York

16. Fisher N 1995 Statistical Analysis of Circular Data. Cambridge University Press, New York

17. Zar J 1999 Biostatistical Analysis. Prentice-Hall, Upper Saddle River, NJ

18. Kelso JA, Scholz JP, Schoner G 1986 Nonequilibrium phase transitions in coordinated biological motion: critical fluctuations. Phys Lett A 118:279-284

19. Scholz JP, Kelso JA 1989 A quantitative approach to understanding the formation and change of coordinated movement patterns. J Mot Behav 21:122-144

20. Koenig JS, Davies AM, Thach BT 1990 Coordination of breathing, sucking, and swallowing during bottle feedings in human infants. J Appl Physiol 69:1623-1629

21. Hammerman C, Kaplan M 1995 Oxygen saturation during and after feeding in healthy term infants. Biol Neonate 67:94-99

22. Mathew OP 1988 Respiratory control during nipple feeding in preterm infants. Pediatr Pulmonol 5:220-224

23. American Academy of Pediatrics 2005 Breastfeeding and the use of human milk. Policy statement. Pediatrics 115:496-506. 